\title{
A Comparative Assessment of the Navy's Future Naval Capabilities (FNC) Process and Joint Staff Capability Gap Assessment Process as Related to Pacific Command's (PACOM) Integrated Priority List Submission
}

\section{Technical Report}

By: Jaime Frittman, Sibel McGee, PhD, John Yuhas

13 December 2012

Analytic Services Inc. (ANSER)

2900 South Quincy Street, Suite 800

Arlington, VA 22206 


\section{Report Documentation Page}

Form Approved

OMB No. 0704-0188

Public reporting burden for the collection of information is estimated to average 1 hour per response, including the time for reviewing instructions, searching existing data sources, gathering and maintaining the data needed, and completing and reviewing the collection of information. Send comments regarding this burden estimate or any other aspect of this collection of information,

including suggestions for reducing this burden, to Washington Headquarters Services, Directorate for Information Operations and Reports, 1215 Jefferson Davis Highway, Suite 1204, Arlington

VA 22202-4302. Respondents should be aware that notwithstanding any other provision of law, no person shall be subject to a penalty for failing to comply with a collection of information if it

does not display a currently valid OMB control number.

1. REPORT DATE

13 DEC 2012
2. REPORT TYPE
3. DATES COVERED

00-00-2012 to 00-00-2012

4. TITLE AND SUBTITLE

A Comparative Assessment of the Navy's Future Naval Capabilities

(FNC) Process and Joint Staff Capability Gap Assessment Process as

Related to Pacific Command's (PACOM) Integrated Priority List

Submission

6. AUTHOR(S)

7. PERFORMING ORGANIZATION NAME(S) AND ADDRESS(ES)

Analytic Services Inc. (ANSER),2900 South Quincy Street, Suite

800,Arlington, VA,22206

9. SPONSORING/MONITORING AGENCY NAME(S) AND ADDRESS(ES)

5a. CONTRACT NUMBER

5b. GRANT NUMBER

5c. PROGRAM ELEMENT NUMBER

5d. PROJECT NUMBER

5e. TASK NUMBER

5f. WORK UNIT NUMBER

8. PERFORMING ORGANIZATION

REPORT NUMBER

10. SPONSOR/MONITOR'S ACRONYM(S)

11. SPONSOR/MONITOR'S REPORT

$\operatorname{NUMBER}(S)$

12. DISTRIBUTION/AVAILABILITY STATEMENT

Approved for public release; distribution unlimited

13. SUPPLEMENTARY NOTES

14. ABSTRACT

15. SUBJECT TERMS

16. SECURITY CLASSIFICATION OF:

a. REPORT

unclassified

b. ABSTRACT

c. THIS PAGE

unclassified

17. LIMITATION OF ABSTRACT

Same as

Report (SAR)
18. NUMBER

OF PAGES

55 
This Page Intentionally Blank 


\section{Table of Contents}

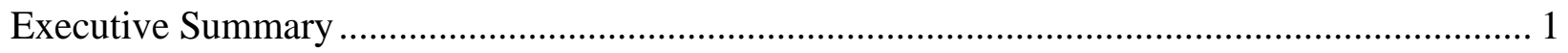

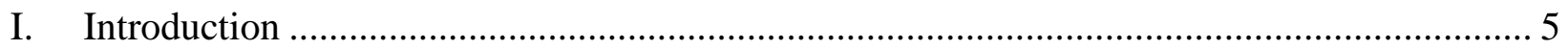

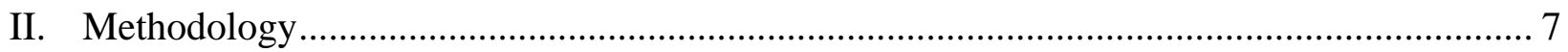

III. Overview of the FNC and Joint Staff CGA/IPL Systems ............................................. 11

IV. The Conceptagon Overview .................................................................................... 14

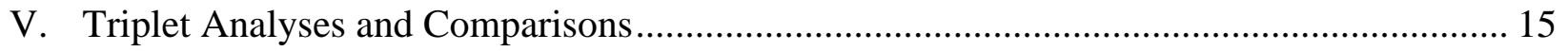

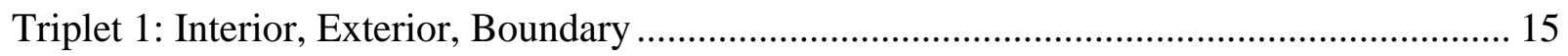

Triplet 2: Wholes, Parts, Relationships ......................................................................... 19

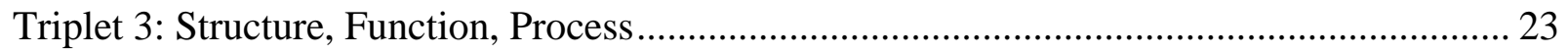

Triplet 4: Inputs, Outputs, Transformations ..................................................................... 28

Triplet 5: Command, Control, Communications ............................................................ 32

Triplet 6: Openness, Hierarchy, Emergence ................................................................... 35

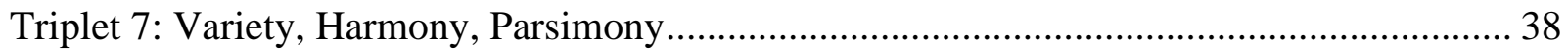

VI. Conclusions, Recommendations, and Strategies for Improvements ................................. 42

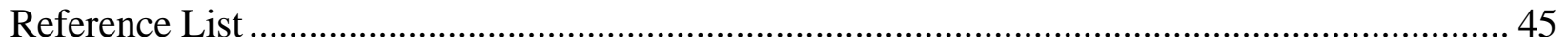

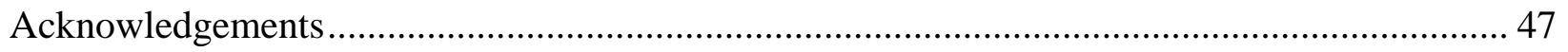

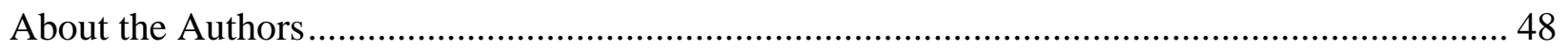


This Page Intentionally Blank 


\section{Synopsis}

This study sought to address whether acquisition is responsive to Combatant Command (COCOM) needs by comparing a responsive system (the Navy’s Future Naval Capabilities [FNC] process) to the Joint Staff (JS)/COCOM process, using Pacific Command (PACOM) as an example. Recognizing different end-states ascribed to both processes, we compared the two processes in terms of gap identification and development—common to both. We concluded that while the JS/PACOM system attempts to be responsive, current practices (e.g., informal tracking mechanisms) prevent an authoritative evaluation of responsiveness. We provided key insights into FNC practices that enhance responsiveness and offered recommendations for improving the JS/PACOM process. 
This Page Intentionally Blank 


\section{Executive Summary}

This report presents the results of our research and analyses on current and future operational capability gap development and acquisition practices in the United States Navy and the Combatant Commands (COCOMs), as exemplified by the Pacific Command (PACOM). Leveraging key stakeholder interviews and using a systems thinking framework known as the Conceptagon, we investigated and assessed the Navy's Future Naval Capabilities (FNC) process and the Joint Staff Capability Gap Assessment (CGA) process as it applies to the annual submission of PACOM's Integrated Priority List (IPL) of capability needs. The study approached both processes as systems and identified and explored their critical systemic attributes such as parts, relationships, boundaries, governance mechanisms/structures, key processes, transformations, stakeholders and missions, to name a few. Based on this assessment, we conducted a structured and systematic comparison of the two processes to identify good practices and favorable dynamics that are likely to reinforce the desired outcome, which-for our purposes-is defined as the resolution of capability gaps and, ultimately, deployment of needed capabilities to the warfighters. In light of this analysis, we present key insights, explore some problem areas, and discuss possible improvements to these processes.

This study's original research question sought to address whether the acquisition process is responsive to COCOM needs by comparing a very responsive system (the Navy's FNC) to the existing Joint Staff (JS)/COCOM system, using PACOM as an example. Recognizing different endstates ascribed to the FNC and the JS/PACOM processes (i.e., FNC identifies/develops naval gaps and continues to develop/transition solutions to the warfighter; the JS/PACOM process ends with the establishment of final gaps), we compared the two processes in terms of gap 
identification and development—which are common to both processes—noting the significant impact of the integration of solution development activities on outcomes. Upon comparing the two systems, the study team concluded that while the JS/PACOM system attempts to be responsive to PACOM capability needs, current practices (i.e., modification of originally submitted COCOM gaps, ensuing difficulty in matching specific solutions to modified gaps, and informal tracking mechanisms for transition of solutions to warfighters) prevent an authoritative evaluation of the relative responsiveness to COCOM capability needs. In light of this assessment, the study team has provided key insights regarding those practices that enhance FNC process responsiveness and has offered recommendations for incorporating similar practices into the JS/PACOM process.

The bulleted list that follows offers a summary of key insights, along with recommendations and strategies for improvement.

\section{Key Insights Into Effective Practices of the FNC Program}

- Participatory and binding measures (as exemplified by FNC roundtable and technology transition agreements) create a sense of collective process ownership amongst stakeholders who may otherwise have differing interests. This increases the chance of solution resourcing and development.

- Communicating intended outputs and outcomes relative to the gap development process (as done by FNC in roadshows and associated briefing materials) helps to manage stakeholder expectations and inform stakeholder perceptions of success, culminating in improved stakeholder commitment and acceptance of gap development processes and associated fulfillment activities. 
- FNC identification and tracking of gap fulfillment measures (e.g., transition statistics) allows the FNC program to adjust in order to improve performance.

- FNC integration of processes that represent the entire life cycle of a gap (from gap identification through capability deployment to the warfighter) promotes a seamless transition between different phases of the effort, facilitates the flow of required information, and provides for continuity of efforts.

\section{How FNC Practices Could Improve the JS CGA/PACOM IPL Process}

- The JS CGA/PACOM IPL process could expand its boundary to include solution providers. Existing structures (e.g., Functional Capability Boards [FCBs], supporting working groups) can be used to facilitate formal participation by the acquisition community.

- COCOM organizations could receive feedback on JS disposition of gap information. Formal, accountable, ongoing, and two-way communication will facilitate understanding, expectation management, and feedback. JS could establish formal communication mechanisms to provide updates on gap modifications (i.e., merging similar gaps, capability board adjustments) and outcomes.

- Gap and solution progress statistics, collected in coordination with the acquisition and warfighter communities (currently partially tracked by JS and accessible through Knowledge Management and Decision Support [KMDS]), could be documented and published periodically (e.g., annually or bi-annually) and could be actively disseminated to COCOMs and briefed at FCBs and Joint Capability Boards (JCBs).

- These outcome-tracking metrics could be used by both JS and COCOM staffs to inform process improvement efforts. The JS process could establish procedures and update 
Instructions and guidance documents to better define roles and responsibilities with metric and process accountability. 


\section{Introduction}

The Department of Defense's (DoD) annual Integrated Priority List (IPL) process ${ }^{1}$ is an integral part of the Joint Staff's (JS) Capability Gap Assessment (CGA) process, ${ }^{2}$ which, once published, is one input among many considered by defense acquisition communities. This process, however, may result in high-priority IPL capability gap(s) receiving lower priority consideration in the military departments' resource allocation and acquisition decisions. By contrast, the Navy's Future Naval Capabilities (FNC) process is considered by both naval and non-naval audiences to be a remarkably responsive and integrated process that supports naval warfighter needs. This is further substantiated by the FNC process' transition statistics, which demonstrate a record of successfully addressing naval capability gaps through the allocation of resources and establishment of effective schedules for research, development, and acquisition of needed capabilities (Office of Naval Research, 2012).

In this paper, we present a comparative assessment of the Navy's FNC process and the JS CGA/COCOM IPL process as exemplified in the PACOM IPL process. This study acknowledges FNC is an end-to-end process inclusive of solution development and transition, whereas the JS CGA/COCOM IPL process focuses solely on gap identification and assessment with little integration of solution activities. Although this comparison may first appear to be asymmetric, we compared the processes across similar steps that culminate in the shared milestone of establishing final, prioritized gaps (see Figure 1).

\footnotetext{
${ }^{1}$ The IPL process is intended to address the nine Combatant Commands' Joint warfighting needs through identification and prioritization of operational capability gaps.

${ }^{2}$ Results of the CGA process are published through a Joint Requirements Oversight Council Memorandum (JROCM).
} 
Figure 1: Common Milestones of FNC and JS CGA/IPL

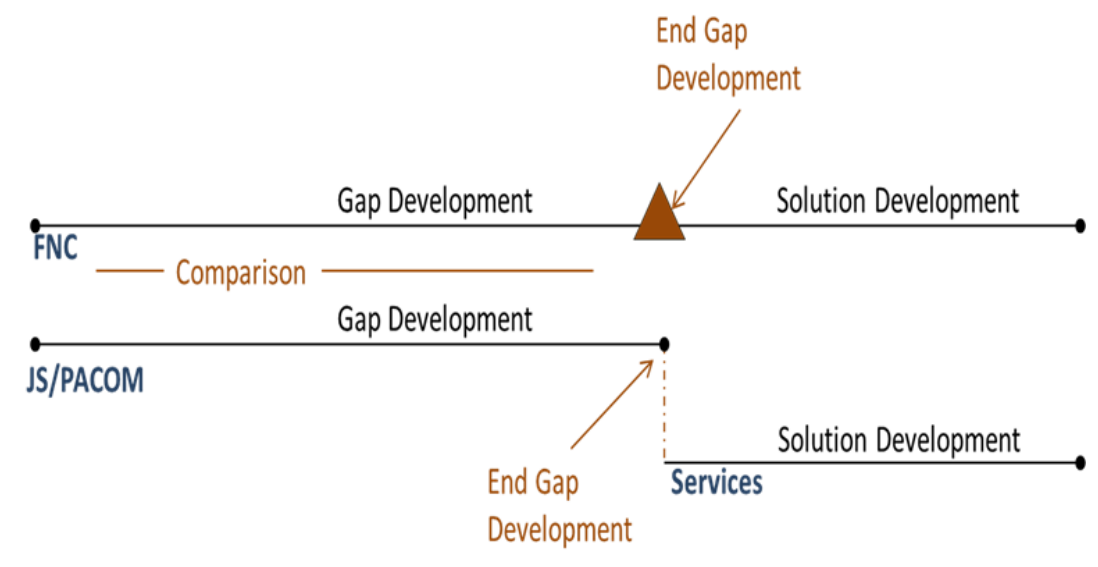

Throughout the course of the study, however, it was frequently noted that integrated solution development activities significantly influenced FNC's gap development activities. As we explain in our conclusions, in FNC, the interaction and mutual feedback between these steps maximizes the overall process’ responsiveness to naval warfighters’ needs. 


\section{Methodology}

\section{A Systems Thinking Approach to Data Compilation and Comparison}

The team chose to approach data analysis using a systems thinking approach, which is particularly useful when assessing soft systems, such as enterprises and processes. In this study, the FNC process and the JS CGA/PACOM IPL development process were viewed as systems, each with constituent parts, united by relationships, working together to achieve a specific purpose.

In particular, the team used the soft systems framework, the Conceptagon (Boardman \& Sauser 2008), to assess the FNC and JS CGA/ PACOM IPL processes. The Conceptagon served as a consistent framework for comparison, standardizing the team's approach to characterizations. It allowed the team to compare seemingly disparate processes across set dimensions of interest. For example, instead of trying to compare the Joint Staff J-8 with the FNC IPT (a difficult comparison at its face value) the team compared across more abstract dimensions such as “actors” and "relationships.” Such dimensions of comparison were used throughout the study to facilitate a consistent and coherent approach to identifying the underlying similarities and differences between the two processes.

\section{Data Collection}

Data and relevant information for this study were collected through a literature review and interviews. To prepare for assessment of FNC, study team members attended the Office of Naval Research’s FNC external training. This half-day course provided instruction on FNC program basics (FNC goals, objectives, and participants) and key program management processes. In 
addition to the half-day course, the team conducted interviews with the FNC Program Director (Steve Smolinski) and members of the director's staff. The team also reviewed Integrated Process Team (IPT) charters, FNC briefings, FNC policy memorandums, and related naval instructions. To prepare for assessment of PACOM's IPL, and its movement through the broader Joint Staff CGA process, the team reviewed PACOM documents such as the Plans to Resources to Outcomes Process (PROP) briefings. The team also conducted interviews with participants in the PACOM IPL process, such as the PACOM Science Advisor and PROP and future capabilities subject-matter experts. To understand what happens once the IPL entries are submitted to the Joint Staff, the team interviewed members of the Joint Staff J-8, Joint Capability Office. The team also reviewed a number of applicable Chairman of the Joint Chiefs of Staff Instructions (CJCSIs). For general awareness, cleared members of the team reviewed additional information, including actual IPL submissions.

\section{Research Questions and Study Scope Adjustments}

The study was initially scoped to investigate whether the acquisition process is responsive to COCOM needs. This was to be accomplished by researching two or more COCOM IPL processes and the solutions that resolved their identified gaps. During the data collection phase, however, the study team recognized that an adjustment to the research question, as well as the scope of the study, was necessary.

To answer the original research question (i.e., Is the acquisition process responsive to COCOM needs?) the team needed to explore four major research areas: (1) how COCOM needs are identified (collection of warfighter statements of operational shortfalls), (2) how operational shortfalls are captured and communicated (development of the COCOM IPL), (3) how IPL 
statements are transformed into Joint Capability Gap statements (development of the Joint Capability Gap Assessment); and (4) how Joint Capability Gaps are resolved (Service efforts to fund and develop solutions). While the fourth research area may hold specific statistics that seemingly answer the question, these statistics are subject to great error if the gaps to which the Services are responding do not accurately reflect COCOM needs. We realized that answering the question was not just a matter of gathering statistics on Service development programs, but also about exploring the gap development process that informs gap resolution efforts. Therefore, we focused on the gap development processes (Areas 1-3) rather than Service solution funding and development (Area 4), which would have been a study in its own right.

In addition, a comprehensive review of gap development processes for multiple COCOMs would require more time and resources than were allotted. As a result, instead of researching multiple COCOMs, which may have yielded a more comprehensive but less detailed analysis, the team conducted an in-depth analysis of a single COCOM. The decision to examine PACOM was made for several reasons: (1) PACOM had a mature gap identification process; (2) PACOM’s process is well documented and the team had access to PACOM briefings, instructions, and materials; (3) the team had personal contacts in the PACOM IPL development process who agreed to participate in interviews. By reducing the number of COCOMs researched, the team was able to provide a holistic view of the overall process to include activities at the JS level. This change provided the team with a more comprehensive view of a COCOM IPL's life cycle.

\section{Limitations of the Study}

Due to the decision to focus on a single COCOM, the resulting recommendations may be less applicable to some COCOMs. Also, information used throughout the study was based on access 
to available documentation and the professional views/perceptions of the individuals

interviewed; as a result, some of the information is subject to personal bias and limited to the experience of those interviewed. Finally, grant conditions required study information to remain unclassified; therefore, specific IPL information is not discussed. 


\section{Overview of the FNC and Joint Staff CGA/IPL Systems}

The following section presents a high-level overview of both the FNC and JS CGA/PACOM IPL processes, and provides grounds for understanding the subsequent Conceptagon assessment.

\section{Future Naval Capabilities}

Initiated in 2002 (Office of Naval Research, n.d.a), the FNC program and the associated process addresses naval (Navy and Marine Corps) gaps with science and technology (S\&T) solutions on an annual basis (Office of Naval Research, 2012). Using 6.2 (i.e., applied research) and 6.3 (i.e., advanced technology development) funding, this program “develop[s] ... quantifiable technology products in response to validated S\&T gaps” within a five-year time frame (Office of Naval Research, 2012). Upon maturation of technology and fulfillment of exit criteria (Office of Naval Research, 2012), the FNC program transitions related products to naval acquisition programs of record "for timely incorporation into platforms, weapons, sensors, and process improvements” (U.S. Naval Research Laboratory, n.d.). ${ }^{3}$ The FNC process is currently organized along "9 pillars of Enabling Capabilities (ECs)” (Office of Naval Research, 2012), each of which "is an aggregate of science and technology that is aligned to an identified warfighting gap or warfighting capability, and it can deliver a distinct, measurable improvement that contributes to closing the corresponding warfighting gap” (Office of Naval Research, n.d.b). ${ }^{4}$

\footnotetext{
${ }^{3}$ The FNC program deals only with products whose technology readiness levels (TRL) are between three and six (Office of Naval Research, 2012).

${ }^{4}$ As explained in the FNC external training (Office of Naval Research, 2012), EC pillars include sea shield, sea strike, sea basing, FORCEnet, naval expeditionary maneuver warfare, capable manpower, force health protection, enterprise and platform enablers, and power and energy.
} 
The FNC program is managed through a collaborative process. Broadly speaking, ${ }^{5}$

OPNAV/HQMC requirements are assessed to identify gaps with S\&T solutions. These gaps are then assigned to related pillars for identification of potential solutions. Integrated Product Teams (IPTs) forward prioritized ECs. These ECs are reviewed, assessed, and approved by the Technical Oversight Group (TOG), a three-star Navy and Marine Corps Board of Directors. Related products begin the development phase with strict conditions for an eventual transition to the warfighter as agreed upon by representatives from requirements/resource communities, science and technology developers, and the acquisition community. The overall program is administered by the Office of Naval Research (ONR).

\section{DoD Joint Staff Capability Gap Assessment /Integrated Priority List Process and Its Employment by Pacific Command}

The annual CGA/IPL process produces a prioritized list of DoD warfighting capability gaps that impact DoD Combatant Command’s (COCOM) execution of operational, contingency, and campaign plans. This process informs the JS CGA, Functional Capability Board (FCB) planning guidance, and development of the Chairman’s Program Assessment (CPA), and analyzes baseline resource allocation priorities for the subsequent years’ IPLs (K. Carlan, personal communication, April 11, 2012; D. Glenister, personal communication, April 26, 2012).

Each COCOM has a different process for generating their input into the DoD Joint Staff CGA/IPL ${ }^{6}$ process. PACOM's employment of the JS CGA/IPL process is comprised of four

\footnotetext{
${ }^{5}$ The following discussion of the FNC process relies on information gathered during FNC training that members of the project team attended at ONR on March 9, 2012, and the PowerPoint slides (Office of Naval Research, 2012) disseminated during the training.

${ }^{6}$ In some cases, we refer to the JS CGA/IPL process as opposed to the JS CGA/PACOM IPL process. This change in nomenclature is intended to capture instances wherein discussion points are likely relevant to multiple COCOMs.
} 
fundamental activities including development, submission, assessment, and validation of capability gaps (K. Carlan, personal communication, April 11, 2012; Carlan, 2011). ${ }^{7}$ (1) The process begins with the identification, organization, and development by PACOM components and sub-Unified Command organizations of capability gaps through a collaboratively facilitated Plans to Resources to Outcomes Process (PROP) within the PACOM J-8 Resources and Assessment Directorate. USF-J, USFK, USARPAC, PACFLT, MARFORPAC, PACAF, SOCPAC, JIATF-West, JPAC and ALCOM are the participating PACOM PROP organizations. The PROP results, used for a number of PACOM purposes, including as an input to the IPL, are reviewed and revised by key O-6 level staff officers, J-code Directors, and the PACOM Deputy Commander. (2) The PACOM Commander then prioritizes, approves, and submits the PACOM IPL to the Joint Staff in support of the JS J-8 Force Structure, Resources, and Assessment Directorate CGA process, and informally to the Under Secretary of Defense (Acquisition, Technology, and Logistics; USD[AT\&L]). (3) JS J-8 conducts a CGA of all nine COCOM IPLs including PACOM; (K. Duffy \& D. Glenister, personal communication, April 16, 2012). ${ }^{8}$ The assessment process includes review and analysis of IPL capability gaps by nine FCBs, which determine warfighting relevance and result in recommended capability solutions and funding. This process produces and submits a J-8-recommended prioritized list of capability gaps and associated solutions to the Joint Capability Board (JCB). The JCB reviews and recommends the capability gap list to the Joint Requirements Oversight Council (JROC). 4) The JROC validates capability gaps and publishes a JROC Memorandum (JROCM) CGA, which is distributed to the USD(AT\&L) and the Services for action on capability solutions acquisition and fielding.

\footnotetext{
${ }^{7}$ All discussion on PACOM IPL activities, including PROP, is based on personal interviews with Kit Carlan and Ken Bruner of PACOM, and PowerPoint slides dated March 23, 2011 that were prepared by Kit Carlan.

${ }^{8}$ The information about JS CGA process and details are based on interviews with Air Force Col. Keith Duffy of JS and Navy CAPT. Dave Glenister of JS.
} 


\section{The Conceptagon Overview}

The Conceptagon framework (Boardman \& Sauser, 2008) aids analysts in conceptualizing and characterizing a system. Boardman and Sauser (2008) define a system's attributes in seven easyto-remember sets, called “triplets.” These triplets and our understanding of them are explained in the following list:

- Interior, exterior, boundary - This triplet describes the perimeter that separates entities that comprise the system from entities outside the system's control.

- Wholes, parts, relationships - This triplet requires identification of the system at hand, the constituent pieces, and the relationships that bind those pieces together.

- Structure, function, process - This triplet identifies the key composition, arrangement, or organization (structures) a system employs to support key activities (processes) necessary to produce the desired system behavior (function).

- Inputs, outputs, transformations-This triplet identifies items coming into the system (inputs) and items exiting the system (outputs) as products or deliverables. The triplet also identifies the change (i.e., transformation) that converts inputs to outputs.

- Command, control, communication-This triplet explores the system's governance structures and control mechanism, and takes into account communication feedbacks and stovepipes.

- Openness, hierarchy, emergence-This triplet investigates a system's ability to accept inputs from the exterior environment and to absorb and accommodate new components (openness), to reconfigure itself in light of new requirements (hierarchy), and to respond to and manage unexpected behaviors produced by such changes (emergence).

- Variety, harmony, parsimony - This triplet refers to the system's design balance, assessing if the system has "too much" or "too little" of anything. 


\section{Triplet Analyses and Comparisons}

\section{Triplet 1: Interior, Exterior, Boundary}

\section{Navy's FNC Process}

There are three pertinent FNC boundaries: program specification, temporal, and stakeholder. The first boundary is established in reference to program specifications, which provide that the FNC system works only with 6.2 and 6.3 funding and is only authorized to handle naval gaps with S\&T solutions of Technology Readiness Level (TRL) 3 to 6 (Office of Naval Research, 2012). The second boundary defines what is within and beyond the system from a temporal angle as portrayed by Figure 2. Accordingly, operational requirements definition takes place prior to the FNC process. As explained in the FNC training provided on March 9, 2012, the FNC process starts with development of S\&T gaps by the Chief of Naval Operations and Headquarters, Marine Corps staff and continues as follows: ONR responds to the gaps by proposing ECs, an aggregate of one or more technology products aimed at closing or mitigating these gaps (S. Smolinski, personal communication, November 29, 2012). Transition of matured S\&T products into the acquisition POR is also within the bounds of the FNC program. Integration to the acquisition POR, however, happens subsequent to the FNC process (Office of Naval Research, 2012). Finally, the third boundary defines stakeholders who have influence on the FNC system. For example, those stakeholders that actively take part within the FNC process involve IPTs, the TOG, TOG working groups (WGs), resource sponsors, acquisition sponsors, and S\&T developers. Some of the passive FNC stakeholders include OPNAV, FCC/MCCDC, naval warfighters, COCOMs, and industry. 
Figure 2: FNC Temporal Boundaries

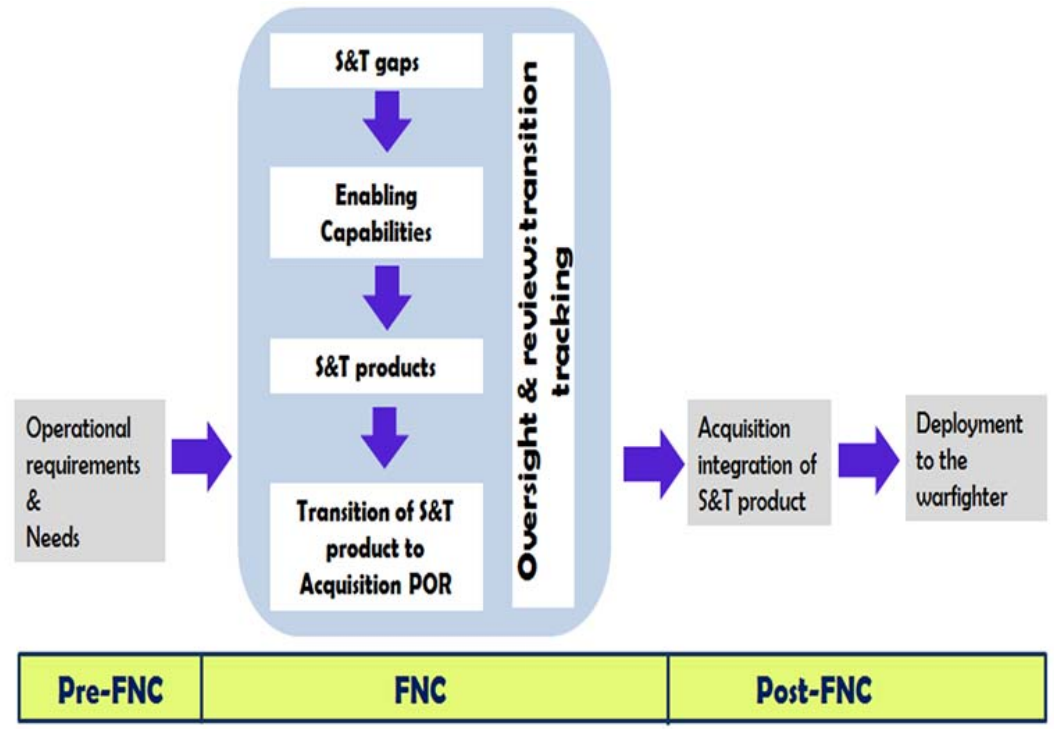

The FNC boundaries can be characterized as semi-porous, presenting degrees of openness across elements, actors, and issues. Acquisition sponsors, resource sponsors, and S\&T developers leverage system flexibility to reach out to and bring in actors from the external environment. This is not to say that the FNC boundaries allow for complete permeability. The program specifications are clear and firm (e.g., non-S\&T gaps are outside the authorization, budget has a clear mandate) and set structures with clear membership descriptions indicate that membership is not ad hoc and does not change over time. The FNC process has well-defined boundaries.

\section{Joint Staff CGA/PACOM IPL Process}

When we consider what is within and beyond the JS CGA/PACOM IPL process, two boundaries appear particularly relevant: conceptual/temporal and stakeholder. According to the conceptual/temporal boundaries (Figure 3), PACOM warfighters provide requirements into the PROP process, which generates PACOM capability gaps/shortfalls for the PACOM Deputy Commander’s review and assessment (K. Carlan, personal communication, December 10, 2012). The PACOM Commander approves and submits the final PACOM IPL to the JS CGA process. 
After going through several steps within this process, a JROCM is issued and conveyed to the COCOMs, the Services, and the OSD offices.

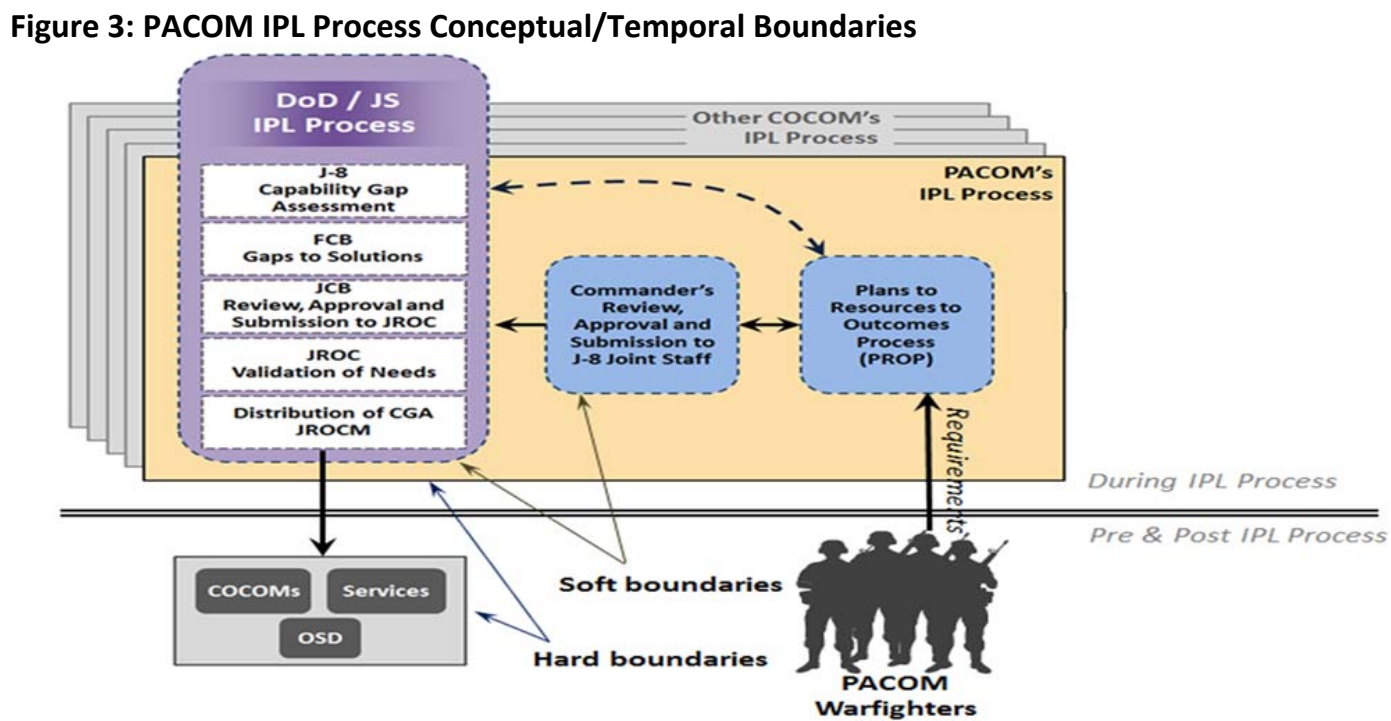

The second boundary applicable to the PACOM IPL Process is the stakeholder boundaries. Active stakeholders include the PACOM Commander, PACOM components, and J-code Directors, JS Functional Capability Board, JS Joint Capability Board, JROC, and JS J-8 (K. Carlan, personal communication, April 11, 2012; D. Glenister, personal communication, April 16, 2012). Some of the passive stakeholders include other COCOMs, the Services, defense agencies, combat support agencies, and inter-agencies. It is important to note that the majority of the Joint Staff CGA/PACOM IPL process stakeholders interact with but are not necessarily controlled by PACOM.

The JS CGA/PACOM IPL process is characterized by mixed boundaries. The boundary between the PROP process and JS-led CGA process is semi-porous: While interaction between the PROP and the PACOM Commander is two way, there are no formally mandated feedbacks subsequent to PACOM’s IPL submission (D. Glenister, personal communication, November 26, 2012). PACOM does not control the JS-led CGA process. The outer boundaries of the JS 
CGA/PACOM IPL process present different levels of openness. At the entry point, the boundary is soft and semi-porous, allowing interaction between the PACOM warfighter and the PROP (K. Carlan, personal communication, April 11, 2012, December 10, 2012). At the exit point, where the JROCM is completed and distributed to the COCOMs, the Services, and the OSD offices, the boundary is hard with no two-way interaction.

\section{Comparative Assessment}

A comparison of programmatic boundaries indicates that while the FNC process has clear programmatic boundaries with specific funding lines and approved types of actionable gaps (e.g., Naval S\&T gaps with solutions at set TRLs), the JS CGA/IPL process does not have an associated type of funding approved/authorized and is expected to address any gap that is deemed significant.

Similarly, comparison of the temporal boundary reveals that even though both processes have a three-phase life cycle (pre, during, and post; see Figures 2 and 3), they differ in system goals. Unlike the FNC system, which is designed to deal with the full gap-to-solution life cycle, the JS CGA/PACOM IPL process is designed to deal with only capability gaps.

Finally, stakeholder boundaries show that the FNC process has a more comprehensive approach to stakeholder participation in line with its full life-cycle process. Unlike the JS CGA/PACOM IPL process, the FNC process includes not only operators, but also resourcers and developers. In addition, all FNC stakeholders are bound by both organization (i.e., single naval command) and process, which ensures a unified organizational vision and direct accountability. The JS

CGA/PACOM IPL process, on the other hand, predominately involves representatives from the 
operational communities (with limited input from the acquisition community) who are bound by process. These stakeholders — controlled by multiple command authority-have competing visions and distributed accountability. The nature of the boundaries also impacts the quality of stakeholder communications. Even though both processes have semi-porous boundaries, FNC relies on a collaborative information exchange (i.e., two-way communications) while the JS CGA/PACOM IPL process involves predominantly one-way information delivery.

\section{Key Insights}

Consequences resulting from differences in system boundaries of these two processes include the following:

- $\quad$ The FNC process is designed to maximize gap resolution through dedicated programs.

- $\quad$ The JS CGA/ IPL system defines gaps, but is not equipped to buy, develop, and produce solutions.

- $\quad$ Unified command structure allows the FNC system to centralize control, while the JS CGA/PACOM IPL process has distributed and decentralized control.

- $\quad$ Broad program definition (all gaps by all COCOMs) introduces complexity into the JS CGA/PACOM IPL process.

- Collaborative communication enhances shared understanding and expectations amongst FNC stakeholders.

\section{Triplet 2: Wholes, Parts, Relationships}




\section{Navy's FNC Process}

The FNC system is comprised of its mission, stakeholders, S\&T gaps, processes, FNC pillars, Enabling Capabilities (ECs), EC proposals, the S\&T budget, and S\&T products. When these parts and their relationships come together, they form a coherent and meaningful whole - the FNC system. Figure 4 shows how these different parts relate to each other. For example, ECs are made up of S\&T products, which respond to S\&T gaps. Similarly, ECs are organized along the nine FNC pillars.

Figure 4: FNC System Parts and Relationships

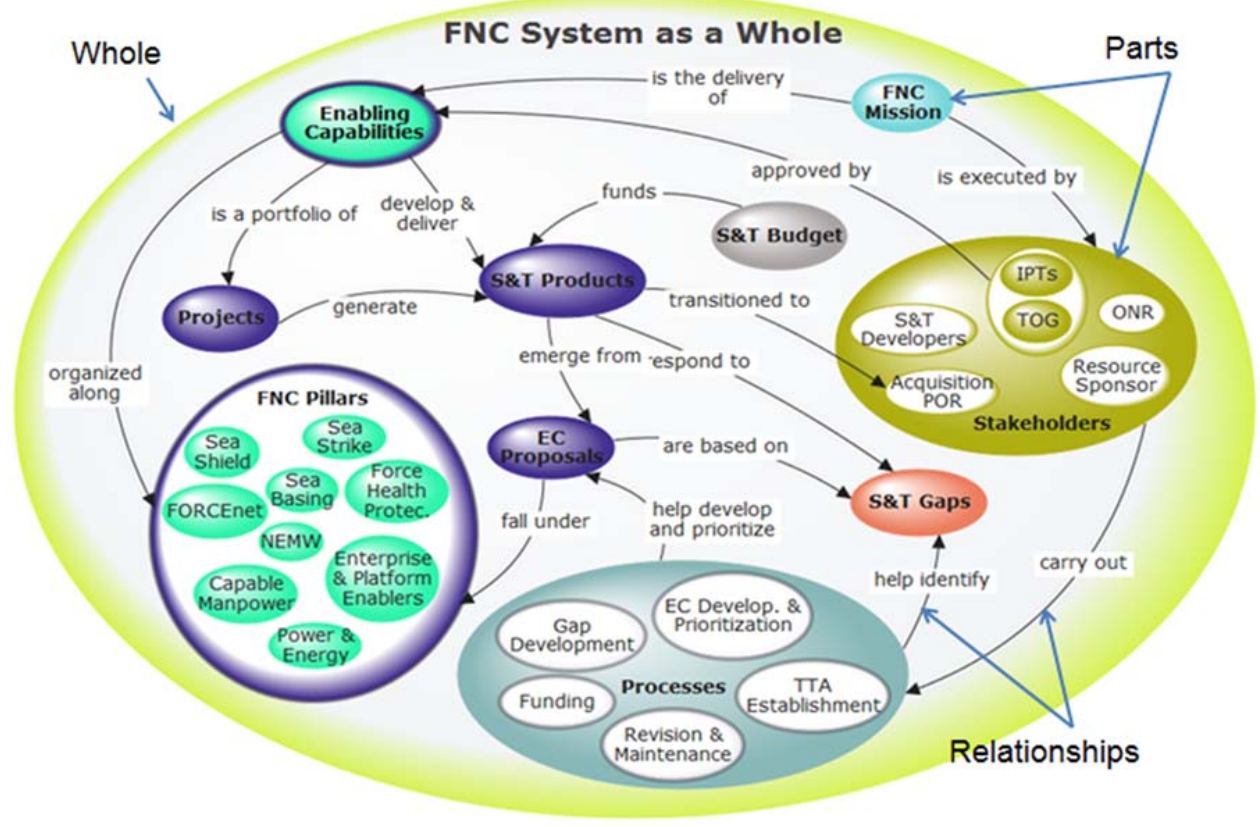

This triplet shows that the FNC process constitutes a well-organized system. Stakeholders have a clear understanding of the parts and the overall system mission. Moreover, the relationships between stakeholders are facilitated by codification of roles and responsibilities. The parts are also knit together with effective flow of information, resources, and activities. As such, they are configured to form a well-connected whole. 


\section{Joint Staff CGA/PACOM IPL Process}

The IPL system as a whole (Figure 5) is made up of stakeholders, processes, PACOM operational shortfalls, PACOM IPL of capability gaps, and Joint capability gaps. Figure 5 maps out the relationships between these parts. As an example, PACOM components identify and prioritize PACOM operational capability gaps, which are the basis for the PACOM IPL.

PACOM IPL capability gaps are then evaluated for and may be incorporated into joint capability gaps.

Figure 5: IPL Process Parts and Relationships

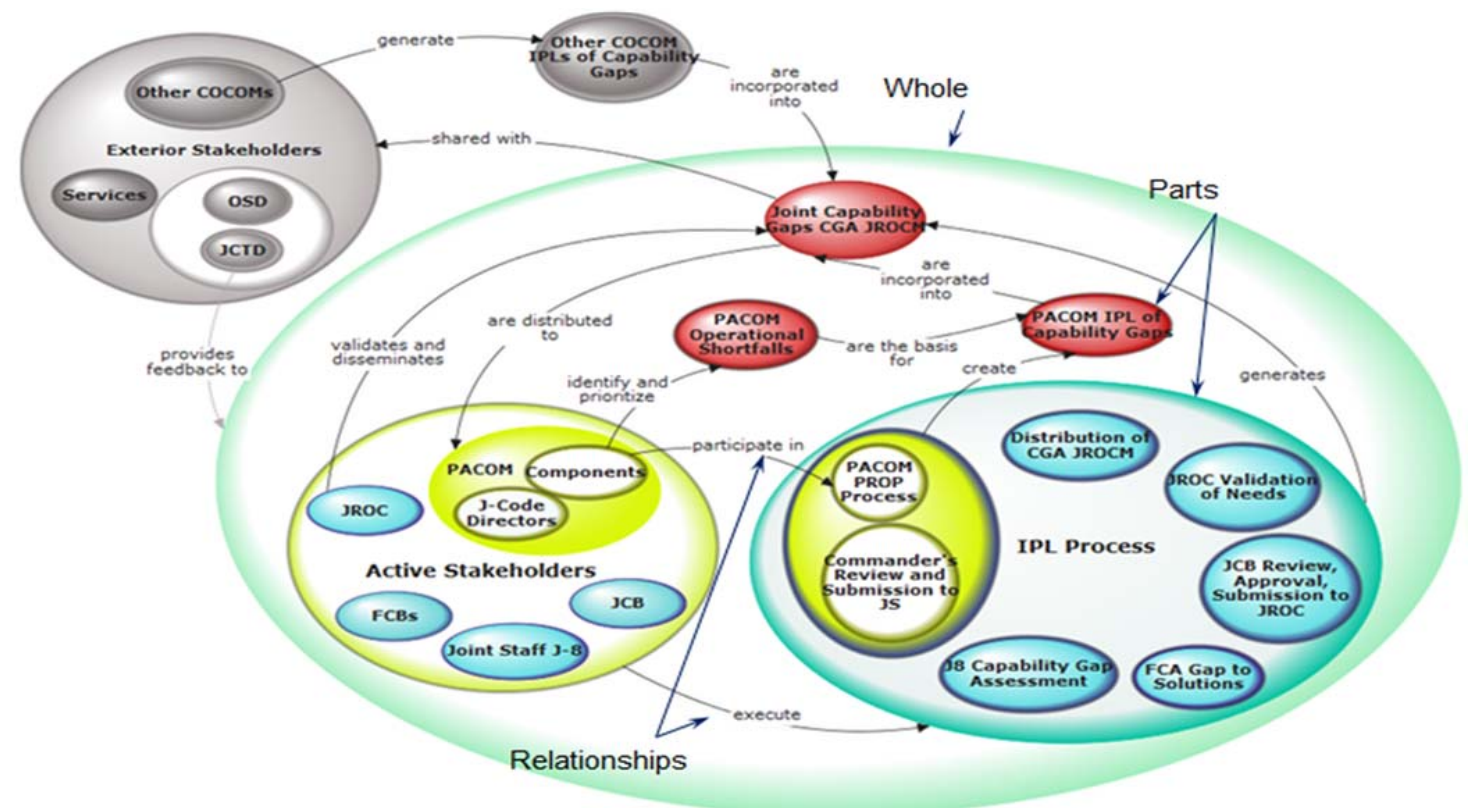

A key observation is that PACOM's IPL development process is dependent on, but does not control, all parts participating in the process. This is significant for two reasons: (1) JS, which does not participate in PACOM's IPL development process, controls the second part of the JS CGA/PACOM IPL process; and (2) Joint capability gap descriptions are affected by other COCOM capability gaps. The diagram also sheds light on the flow of information within PACOM's IPL process. While PROP relationships benefit from a collaborative environment, 
PACOM’s PROP-to-JS relationship is characterized largely through one-way interactions. Additionally, there is limited reporting or feedback on the status of solutions to PACOM's capability gaps; in addition, access to JS tracked information requires that COCOMs pull for information, rather than receive it by way of JS push mechanisms (D. Glenister, personal communications, November 26, 2012).

\section{Comparative Assessment.}

A comparative assessment of parts within the FNC and JS CGA/PACOM IPL systems shows two discrepancies. First, the FNC process can be characterized as a single system, whereas the JS CGA/PACOM IPL process merges two distinct systems—-that is, PROP and CGA (which includes other COCOM gap development processes)—-to create a new system. Second, the FNC process identifies, develops, and pursues specific solutions. The JS CGA/IPL process does not involve specifically designated budget and other process parts that target solution (or product) development (K. Carlan, personal communications, April 11, 2012). Solution development is limited to the FCBs' preliminary investigations on potential solution sets. ${ }^{9}$

A closer look at these two processes reveals a number of differences in underlying communication approaches. FNC supports feedback relationships that inform participants throughout the process and allow changes, as required. The JS CGA/PACOM IPL process, on the other hand, has limited feedback, generally in the form of a briefing to stakeholders about final Joint gaps, with limited opportunity for follow-on changes. In the case of the JS CGA/PACOM IPL process, this communication approach has three implications: (1) It degrades efforts to maintain continuity of effort and intent from the PACOM Commander through the

\footnotetext{
${ }^{9}$ The COCOMs list known on-going efforts (OGEs) and recommended actions/solution, and the FCBs request Service input to review and comment on COCOM input and provide their own input.
} 
final JS outcome, (2) stakeholders may have limited opportunity to influence outcomes and may not fully understand the reasoning behind final decisions, and (3) the ability of the process to self-correct based on process outcomes is limited.

\section{Key Insights}

Some of the important consequences of these differences are listed as follows:

- The JS CGA/PACOM IPL process combines multiple systems and stakeholders, creating a potential for operational strife and inefficiencies. Achieving process integration and cohesiveness requires ongoing, two-way communication, which, currently, is limited.

- FNC solution investigations are more rigorous than those in the JS CGA/PACOM IPL process due to follow-on pursuit of product development.

- Current communication relationships between PACOM, JS, and, ultimately, the Services may lead to different (perhaps conflicting) understandings/expectations amongst stakeholders.

- In the JS CGA/IPL process, current procedures for reporting on solution status may curb shared understanding of how individual COCOM gaps will be resolved.

\section{Triplet 3: Structure, Function, Process}

\section{Navy's FNC Process}

The intended outcome of the FNC process is to identify and validate an annual list of operational capability gaps and to develop solutions that can be transitioned to a program of record. There are five primary organizational structures that participate in the process: nine IPTs, which are organized along nine FNC pillars; the TOG; a Resource Sponsor; Program Executive Office 
Systems Command (PEO/SYSCOM) and S\&T (Office of Naval Research, 2012). Their configuration and participation is determined according to long-standing FNC procedures and practices. Each organization performs a unique set of roles and responsibilities, producing welldefined information and capability solution deliverables.

The FNC process involves seven functions performed by the aforementioned organizations in the following manner, as explained during FNC training (Office of Naval Research, 2012): Subsequent to the review of operational problems, the nine IPTs develop and forward their top three non-prioritized S\&T gaps to the TOG. The TOG reviews and approves the S\&T gaps and forwards them to OPNAV. OPNAV officially promulgates and issues the gaps to ONR for development and prioritization of ECs. EC proposals are developed through collaborative discussions with applicable IPTs. Furthermore, Technology Transition Agreements (TTAs), which serve as integral components of the ECs, are developed by EC project managers. The ECs are then forwarded to the IPTs for overall prioritization. The prioritized ECs are reviewed and consolidated by the TOG Working Group and provided to the TOG for approval. The TOG forwards the ECs to the S\&T Sponsor who then submits the approved ECs as the Sponsor Program Proposal (SPP) to the Chief of Naval Research for implementation through 6.2 and 6.3 S\&T projects. Oversight and review is performed on an ongoing basis through TOG and IPT meetings as well as EC project execution, product development, and the tracking of the transition to the POR and to the warfighter.

\section{Joint Staff CGA/PACOM IPL Process}

The primary intended outcome of the JS CGA/PACOM IPL process is to identify, prioritize, and validate an annual prioritized list of operational capability gaps. It is distributed amongst 
multiple stakeholders who own different stages of the process. The six primary stakeholders of the process are the PACOM PROP components, PACOM Commander, Joint Staff J-8, FCBs, JCB, and JROC. They are configured and linked according to long-standing Joint Staff and PACOM practices. These organizations operate in a sequential, and, at times, interdependent manner to annually assess COCOM capability gaps along with a preliminary review of solutions. Each organization performs a unique set of roles and responsibilities, which can contribute at times to fragmentation between Joint Staff and PACOM stakeholders.

Seven IPL functions are performed by these organizations in the following manner (as confirmed in personal communications with K. Carlan, April 11, 2012): The PACOM PROP organizations, which include USF-J, USFK, USARPAC, PACFLT, MARFORPAC, PACAF, SOCPAC, JIATF-West, JPAC, and ALCOM, identify, organize, and prioritize their organization's operational capability gaps and shortfalls. Operational officers have responsibility to present and convey their organization's warfighting needs through collaborative data entry and dialogue. Commander's guidance, Defense Readiness Review System (DRRS) deficiencies, mission and associated operational context, operational requirements, and available technology are all examples of informational inputs to the PROP. PACOM J-8 facilitates the PROP and performs joint analysis across COCOM missions and operations. It leads the formulation, development, and coordination of the PACOM IPL. Utilizing PROP data, along with other current efforts (e.g., Issue Nominations, DRRS deficiencies, rebalance initiatives), PACOM J-8 prepares and submits the recommended IPL to PACOM key O-6-level staff officers, J-code Directors, and the PACOM Deputy Commander for review, and incorporates feedback in preparation for submission to the PACOM Commander. The IPL is submitted to the PACOM Commander for comment and approval. The approved IPL is submitted to Joint Staff J-8 through the 
Comprehensive Joint Assessment (CJA). The JS J-8 is responsible for conducting the annual capability gap assessment, which is guided by national military strategy and informed by IPLs and other warfighting operational needs. The IPL capability gaps are further defined, taking into account similarities and differences across the COCOMs, and thereby creating a greater overall number of gaps beyond the top 90 submitted by the nine COCOMs. J-8 selectively distributes the capability gaps to the nine FCBs for review. The FCBs are responsible for assessing and confirming the relevance of the capability gaps and identifying preliminary capability solutions, if known and available. The FCBs facilitate collaborative information exchange across the Services, the OSD, other non-Service organizations, and COCOMs. FCB results are conveyed to the J-8 through purple slides and quad charts. The JS J-8 develops the recommended list of prioritized capability gaps for review by the JCB. The JCB reviews them and establishes the recommended prioritized list of capability gaps with programmatic recommendations and associated military and strategic risk, and submits them to the JROC. The JROC validates the capability gaps for the DoD, and publishes a JROCM CGA, which is then distributed to the USD(AT\&L) and the Services for capability solutions acquisition and transition to the COCOMs.

\section{Comparative Assessment}

Both the FNC and JS CGA/PACOM IPL processes are driven by a number of informational inputs such as warfighting requirements and operational plans. The processes vary in their final outcomes, whereby technology transition to programs of record is the tracked and measured outcome of the FNC process, while validated DoD capability gaps are the final outcome of the JS CGA/PACOM IPL process. 
Designed for a single Service, the FNC process is a cohesive and well-integrated process. It is employed exclusively through the naval chain of command and the associated procedure. By contrast, the JS CGA/PACOM IPL process is divided along functions executed by the Joint Staff, and multiple COCOM and Service stakeholders, who are guided by varying interests, needs, and resources. As such, process accountability, traceability, and measures of success are inevitably different within each process. FNC activities are comprehensive; FNC represents a “closed loop" approach to the process, enabling measurable outcomes. In contrast, it is difficult for JS CGA/ PACOM IPL stakeholders to quantify how many, and to what degree, gaps are resolved as a result of the JS CGA/IPL process. Furthermore, unlike in the FNC process, in the JS CGA/PACOM IPL process, PACOM capability gaps can lose specificity, be marginalized, or may be disregarded.

\section{Key Insights}

Some important consequences of these differences are listed as follows:

- The way COCOM gaps evolve during the JS CGA/PACOM IPL process results in difficulty tracking program success as capability gaps and capability results are difficult to connect.

- As a solution- and product-driven process, FNC establishes a robust and transparent connection between gaps and solutions, which facilitates tracking program success.

- The JS CGA/IPL process is designed to focus on gap development and validation; as such, it only tangentially addresses gap-to-solution outcomes.

- The hard boundary between capability gap assessment and solutions may limit JS CGA/PACOM IPL stakeholder access to information on program status and results. 


\section{Triplet 4: Inputs, Outputs, Transformations}

\section{Navy's FNC Process}

The FNC process is designed to respond strictly to naval gaps as guided by naval requirements, sources, and mission. Considering all the activities that are taking place within the FNC system, two different transformations are noted: "Requirements to Products” and "Disparate Perspectives to Collective Ownership” (Figure 6). "Requirements to Products” is a systems engineering transformation and, as such, is readily visible with a tangible output. FNC employs a full systems engineering life cycle, creating a seamless, transparent, and traceable transformation from gap to capability. The transition of enabling capabilities from FNC is tracked from early development activities through delivery to the naval warfighter. In this transformation, requirements are turned into products to be transitioned to Acquisition POR. These products are eventually deployed to the warfighter to resolve a capability gap. Clearly, this transformation is critical for the mission of the FNC program and the broader transformation of requirements to naval capabilities.

The second transformation of "Disparate Perspectives to Collective Ownership" is perhaps less noticeable but very critical in making the FNC process a successful one. It is concerned with the evolution of attitudes and a culture of commitment amongst disparate stakeholders. The FNC process has institutionalized mechanisms to create a collaborative environment, establish clear roles and responsibilities, and reinforce a culture of collaboration and accountability. Collaborative participation platforms, TTAs, and Transition Commitment Levels (TCLs) all commit stakeholders to clearly and explicitly stated role assignments, transforming functional area biases of requirements/acquisition communities into an integrated viewpoint and a shared mission amongst distinct stakeholders. This collective understanding of responsibility, 
accountability, and ownership, in turn, enables the "Requirements to Products" transformation referenced previously.

Figure 6: FNC Transformations

Disparate Perspectives to Collective Ownership Transformation

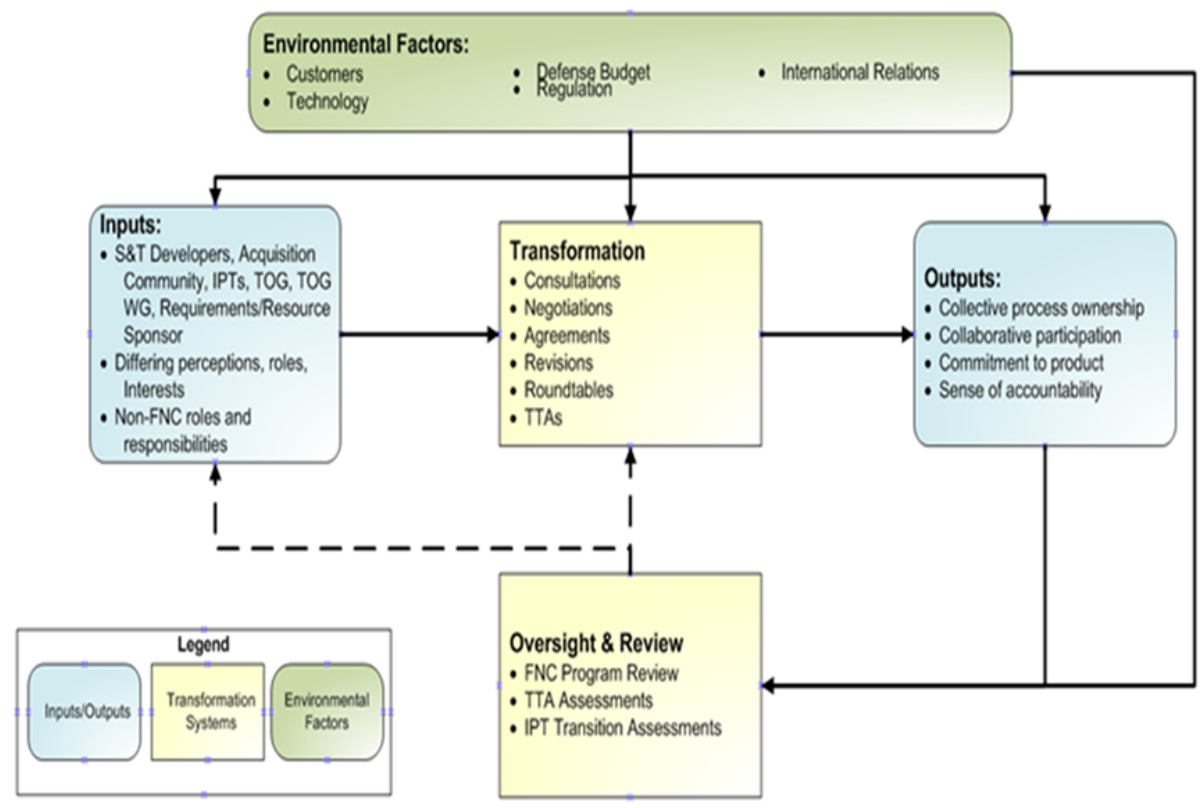

\section{Joint Staff CGA/PACOM IPL Process}

The JS CGA/PACOM IPL process is designed to consider all COCOMs' needs/gaps, each serving as an input to the JS CGA process. Therefore, diverse COCOM capability gaps (determined by their varying missions) are considered and prioritized.

There are three sequential transformations for gap characterization identified within the JS CGA/PACOM IPL process. The first transformation, "PACOM Requirements to PACOM Gaps,” takes place within PROP. Based on PACOM's mission, existing capabilities, available technology, and lessons learned, PACOM components consider PACOM requirements and provide the PACOM Deputy Commander with capability shortfalls/gaps. During the next 
transformation, “PACOM capability gaps to PACOM IPL,” the PACOM Commander evaluates PACOM gaps and issues the final PACOM IPL. Through the third transformation, "PACOM IPL to JROCM CGA,” the PACOM IPL is merged with other inputs (e.g., other COCOM IPLs, national military strategy, etc.) to produce joint capability gaps that are identified and prioritized in the JROCM CGA.

The application of this triplet to the JS CGA/PACOM IPL process brings to the fore the fact that out of the three transformations, PACOM controls only the first two, which results in the PACOM IPL. The third transformation is controlled by JS. Since this transformation is designed to integrate and prioritize DoD-wide capability gaps, it takes into account all COCOMs’ gaps when producing final capability gap descriptions. As a result, PACOM’s IPL is influenced by other COCOMs’ capability gaps and their priorities.

\section{Comparative Assessments}

As opposed to the FNC system, which is more narrowly focused on naval-only gaps, the JS CGA/PACOM IPL process is designed to address DoD-wide capability needs and consider all COCOM capability gaps. As such, a diverse range of missions, actors, requirements, and procedures feeds into the JS process.

A comparison of the FNC and JS CGA/PACOM IPL process transformations shows that FNC measures of success include not only gap-to-solution considerations, but also, product transition to warfighter. The JS CGA/PACOM IPL process, on the other hand, does not include a gap to solution transformation. Additionally, the JS CGA/PACOM IPL system does not have a formal mandated tracking mechanism linking JROCM outputs to solution outcomes. Another difference 
between the two processes is that FNC transforms functional area biases of the requirements/acquisition community into a shared understanding and set of collaborative relationships amongst stakeholders. While the JS CGA/PACOM IPL process does leverage working groups and IPTs as consensus-building platforms, it does not have formal and binding agreements for reconciling varying organizational cultures and norms, even though it serves a more diverse set of clients and needs.

\section{Key Insights}

These differences have several implications for the two processes’ performances:

- PACOM capability gap prioritization may be marginalized due to other COCOM higher priority capability gaps. Similarly, PACOM capability gaps may lose theater operational specificity when/if they are merged with other related COCOM capability gaps.

- Due to the impact of multiple COCOM gaps on joint capability gaps, it is difficult to effectively link individual IPL inputs to joint CGA outputs.

- There is limited ability to effectively track and measure the overall transition success in JSled IPL transformation.

- Cultural transformation in the FNC system enables agreement, shared ownership, and direct accountability.

- FNC outcomes resonate with stakeholders as they produce tangible products as an output.

- By nature of the JS CGA/PACOM IPL process, stakeholder definitions of successful transformation may vary—system transformation may be successful, but it does not mean all stakeholders win. 


\section{Triplet 5: Command, Control, Communications}

\section{Navy's FNC Process}

Command of the FNC process is implemented through ownership and decision-making organizations. The S\&T Corporate Board owns responsibility for the FNC program and establishes policy as well as the participating organizations' roles and responsibilities. The nine IPTs, TOG, and Chief of Naval Research (CNR) have decision-making authorities for the execution of the process, production of the S\&T gaps, and development and transitioning of the ECs and solutions to the POR for operational use. Control of the process, organizational behaviors, and rules of engagement are built-in mechanisms at both the macro and micro levels. DoD 5000.02 (USD[AT\&L], 2008), naval requirements, and the nine FNC pillars provide overarching macro control guidance. The TOG Charter, FNC business rules, TTAs (including TRLs and TCLs), project plans, staff training, and transition metrics are micro level controls to manage and track performance, and to support decision-making throughout the process life cycle.

The FNC process promotes and facilitates formal and informal collaborative information sharing. Closed-loop communications facilitate accountability and provide clear measurable data on outcomes. Information flows through organized coordination and feedback mechanisms with mandatory participation requirements for all stakeholders. Scheduled IPT, TOG, and TOG working group meetings and informal information exchanges enable stakeholders and decisionmakers to assess and track key S\&T gaps and ECs, and to transition capability solutions to the POR for operational use. Dual-hatted IPT co-chairs also facilitate information sharing and general situational awareness through both formal and informal channels as they move between 
FNC and non-FNC worlds. Documentation of the gaps, ECs, project plans, TTAs, and transition reports are established, maintained, and shared across the FNC organizations.

\section{Joint Staff CGA/PACOM IPL Process}

The JS CGA/PACOM IPL process is not owned or governed by a single organization and its stakeholders. JS owns responsibility for the DoD CGA process including the joint capability gap assessment and the JROC validation. PACOM is responsible for developing, approving, and submitting the PACOM IPL. The JROC, JCB, JS J-8, and PACOM Commander have critical decision-making power. As part of their roles and responsibilities, they make decisions to execute the process and to develop and validate capability gaps. FCBs, FCB working groups, and PROP organizations provide prioritized recommendations to the process. There are limited control mechanisms within the JS CGA/PACOM IPL process to facilitate whole-process integration. Formal control is limited due to the absence of such aspects as standard protocols or measures of collective ownership and accountability. The DoD- and PACOM-level controlling guidance includes Defense Planning and Programming Guidance (DPPG); CJCSI 8501.01A (CJCS, 2004), 3110.01G (CJCS, 2008), 3170.01H (CJCS, 2012); JS guidance on how to compose an IPL; Planning, Programming, Budgeting and Execution System (PPBES); Pacific Theater Strategy (1 $1^{\text {st }}$ edition), and instructions for PACOM’s PROP (USPACOM, 2011). ${ }^{10}$

Communications take place predominantly in the form of one-way information delivery. They are performed through written as well as verbal information exchange mediums. The CGA, IPL, purple slides, quad charts, and the JROCM Capability Gap Assessment are classified documents

\footnotetext{
${ }^{10}$ The DPPG, PPBES and JS provide guidance for IPL development each fiscal year. Access to these documents may be restricted to those involved in related planning and resourcing activities. As such, citations are only provided for documents which are known to be publically available. Furthermore these documents were not used to create this report, but rather, serve as examples of documents used by COCOMs for IPL development activities.
} 
that communicate assessed, prioritized, and validated capability gaps. The JROC, JCB, FCBs and working groups, J-8 Worldwide Conference, and PACOM PROP serve as platforms that enable dialogue and discussions across operational and programmatic representatives.

\section{Comparative Assessment}

FNC maintains command of the full life cycle of the process and the associated decision-making since the governance framework that is in place is holistic, integrated, and owned by a single organization. The JS CGA/PACOM IPL process is, on the other hand, governed through multiple separate and independent command structures. PACOM owns its PACOM IPL process as evidenced by the Commander’s approval and submission of the IPL. Thereafter, the JS governs the process for the DoD and all COCOMs. FNC is controlled by macro-level USN guidance and policies and a micro view of instructions, as well as roles and responsibilities. The JS CGA/PACOM IPL process relies mainly on macro command-level guidance driven by DoDwide needs and demands.

Both processes provide opportunities for collaboration and coordination. The JS capability gap assessment is, nonetheless, not dependent on COCOM participation. As such, COCOM capability gaps may be marginalized. Further, COCOM participation is informed by real or perceived limited return on time investment. FNC stakeholders have a clear understanding of the FNC life cycle process and are provided specific updates. COCOMs have diverging perceptions of the process and may be provided more ambiguous information regarding how joint gaps evolve during the cycle and are subsequently fulfilled. FNC offers enhanced accountability through its complete feedback loop that closes the life cycle of a gap by reporting outcomes to stakeholders. JS CGA/PACOM IPL communications are hindered by logistics, practical process 
difficulties, and perhaps most importantly the potential discrepancy between the gap assessments by COCOMs and the DoD.

\section{Key Insights}

These differences between the two processes generate the following implications:

- Centralized and integrated governance and participatory processes that characterize the FNC system provide continuity of process by allowing stakeholder input and agreement throughout the life cycle of the process and by developing shared understanding, joint ownership, and accountability.

- The distributed governance and one-way communications that characterize the JS CGA/PACOM IPL process disrupt the continuity of the process and provide limited opportunities for management of stakeholder perceptions and resulting expectations.

- As a multi-stakeholder process, the JS CGA/PACOM IPL process may need formal integration mechanisms to bring different parts of the process together for shared ownership and enhanced accountability.

- The absence of formal tracking of gap modification and related outcomes limits JS CGA/PACOM IPL process feedback and creates the potential for missed opportunities in adjusting/correcting performance.

\section{Triplet 6: Openness, Hierarchy, Emergence}

Navy FNC Process

The FNC system presents two-way openness throughout its process life cycle: exterior to interior and interior to exterior. Exterior to interior openness is evidenced by the system's ability to 
accept evolving naval requirements/gaps, advances/availabilities of technology, new S\&T developers, and transition venues/methods. The FNC process is also responsive to data on transition to warfighter. Interior to exterior openness is presented by the dual-hatted FNC players' ability to cross into non-FNC domains.

There is some evidence of re-configurability of system hierarchy. For example, "the FNC pillars were reduced from 11 to 5 in 2004" (Goldstein, 2006). Similarly, in 2005 the FNC system "was restructured to align with the pillars of the Chief of Naval Operations' and the Commandant of the Marine Corps' vision for the future—Naval Power 21—and to focus on providing Enabling Capabilities to close warfighting gaps” (Office of Naval Research, n.d.a). ${ }^{11}$

The FNC system accommodates changing circumstances and new process parts; it allows itself to be open to emergent system properties. For example, the transition data influence subsequent FNC processes and performance, which facilitate resource-efficient management of FNC as it permits revision of the process and adjustments in performance based on transition statistics.

\section{Joint Staff CGA/PACOM IPL Process}

The JS CGA/PACOM IPL process is quasi-open and presents mostly one-way openness. Exterior to interior openness of the JS CGA/PACOM IPL process can be seen in its acceptance of evolving requirements and gaps. However, the process is closed to stakeholder feedback subsequent to the issuance of the JROCM. Interior to exterior openness is evidenced by the interior stakeholder communications with the exterior stakeholders.

\footnotetext{
${ }^{11}$ The quote can be found in the Office of Naval Research website as cached by December 9, 2012.
} 
The JS CGA/PACOM IPL process has limited options for change as configuration of system elements appears stable. As a result of limited re-configurability, the system is less versatile and is at risk of critique and perception of ineffectiveness by stakeholders.

\section{Comparative Assessments}

Both FNC and JS CGA/PACOM IPL processes present openness, but the JS CGA/PACOM IPL process' openness terminates subsequent to the JROCM conveyance. Another critical distinction between the two processes is concerned with tracking of transition data. The FNC process is responsive to data on transition to the acquisition POR and eventual deployment of the S\&T product or capability to the fleet. The JS CGA/PACOM IPL process tracks CGA gaps (i.e., COCOM gaps that are accepted as joint gaps), but does not formally track other aspects of the process (i.e., COCOM gap modification and matching of outcome to original COCOM gap).

\section{Key Insights}

Some of the critical consequences that emerge out of the previous discussion include the following:

- Given that there is not a mandated feedback system nor a comprehensive transition tracking system in the JS CGA/PACOM IPL process, stakeholders may pursue what they see as unfilled capability gaps through other means such as JCTDs and JUONS, leading to a potential duplication of efforts and inefficient use of resources.

- The FNC process leverages transition data to modify subsequent FNC process and performance. This may result in a more resource-efficient management of the process. 


\section{Triplet 7: Variety, Harmony, Parsimony ${ }^{12}$}

\section{Navy's FNC Process}

The FNC process introduces an instance of harmony in the way it deals with the tracking of S\&T products and their delivery to the naval warfighter. To achieve harmony, the FNC process tracks two important transitions. First, the process tracks the transition of S\&T products to the intended acquisition POR (Office of Naval Research, 2012). As noted in the boundaries discussion, this transition is an immediate output of the FNC process, and is, therefore, tracked as a critical success metric. Even so, tracking FNC product transition to the POR does not necessarily provide a complete and accurate assessment of product delivery to the warfighter. For example, the POR itself may never transition to the warfighter due to a number of factors, including program cancellation. Similarly, transitioning products to a POR is not an end in itself; rather, it serves a broader purpose. In this regard, tracking only transition to the POR runs the risk of creating a false sense of S\&T integration success and is, therefore, too parsimonious of a view. To counter this, FNC leverages variety in transition statistics. In addition to tracking transition to the POR, the FNC program tracks POR transition to the warfighter (Office of Naval Research, 2012). Just as tracking transition of products to the POR risks creating a false sense of success, tracking only transition of PORs to the warfighter runs the risk of creating an inaccurate assessment of S\&T product failure. This is primarily because S\&T products are but one of many factors in determining a POR's successful transition. It is the balance of viewing success of direct outputs of the FNC process (S\&T product transition to POR) through the lens of broader

\footnotetext{
${ }^{12}$ The triplet of variety, harmony, and parsimony is probably the most abstract Conceptagon triplet of all. To assess this triplet, the study team attempted to identify instances of well-blended variety and parsimony that resulted in harmony, thus bringing the system in balance. Rather than identifying every instance of variety and every instance of parsimony, the study team sought to identify instances of harmony, as these were believed to indicate practices that enhanced system performance.
} 
naval capability delivery (how and if those products are ultimately used by the warfighter) that brings harmony to the FNC program assessments.

\section{Joint Staff CGA/PACOM IPL Process}

By nature of its service to multiple stakeholders across all COCOMs, the JS CGA/PACOM IPL process is subject to great variety. An instance of variety is found in the number of number-one priority gaps submitted to the Joint Staff. Herein COCOMs submit their top gaps (through each COCOM's IPL) to the Joint Staff (later forwarded to the JROC) for inclusion in the final CGA. Individual COCOM gaps are ranked in priority order preceding submission to the JS J-8. This process is conducive to producing much variety in the number of incoming number-one priority gaps, as all nine COCOMs submit a gap designated as number one. The JS J-8 receives the ranked gaps from each of the COCOMs and then works to combine them into a new, consolidated list of prioritized gaps. This consolidation is done so that the DoD can work from a single list of prioritized defense gaps. The process is also used to reduce duplication of gaps and to combine similar gaps. The JS J-8 list is then forwarded through the FCBs and JCB, wherein each group reviews and reorganizes (as required) the list of prioritized gaps prior to final review and approval by the JROC. The passing of the list through many review boards also introduces variety in the process.

While the processes of combining, accepting, rejecting, realigning, and reorganizing COCOM gaps into a single finalized list of joint capability gaps does introduce parsimony (in the creation of a consolidated list), it does not necessarily bring about harmony. By requesting a ranked list of gaps from individual COCOMs, the process inadvertently sets an expectation that the numberone gaps should all receive top priority in the final list. However this may not be the case as one 
COCOM's top 10 needs may make the final list, while another COCOM may not see any of its submitted gaps reflected in the final CGA. Furthermore, the process of homogenizing many seemingly similar gaps into one broad gap statement may create a gap so broad that eventual solutions fail to meet the original need of the submitting COCOM(s). Additionally, variety in the number of reviews as well as groups responsible for approving the CGA fragments the ownership process.

\section{Comparative Assessment}

Both the FNC and Joint Staff processes accept gap statements from various groups (IPTs and COCOMs, respectively); however, by consolidating and reprioritizing COCOM-submitted number-one needs, the Joint Staff process may result in stakeholder frustration. The FNC process benefits from a more direct coordination process as opposed to the Joint Staff process where gaps must travel through many working groups and boards. With each change of hands in the Joint Staff process, gaps are further homogenized, refined, and altered. This extended process of shaping capability gaps can result in gaps that, in some cases, only marginally reflect the originally submitted COCOM need.

\section{Key Insights}

There are two major consequences and their associated ripple effects resulting from these instances of variety, harmony, and parsimony:

- The FNC process achieves harmony at multiple stages of the process through its organizational structure and continuous planning for final transition to the warfighter. 
- Fragmentation of reviewers (through the JS process) and homogenization of COCOM IPLs into joint capability gaps may cause discord leading to general solutions that do not effectively satisfy specific COCOM needs, difficulty in identifying and tracking original COCOM gaps, COCOMs' resubmission of the same gaps in subsequent years. 


\section{Conclusions, Recommendations, and Strategies for Improvements}

\section{Conclusions}

Admittedly, end-states of the two systems (the naval FNC process and the JS CGA/PACOM IPL process) are markedly different; FNC is a gap-to-solution process (including both gap and solution development phases) whereas the JS CGA/PACOM IPL process is solely a gap development process. In addition to varying end-states, the systems differ in the breadth of customers they serve. FNC, a naval program serving naval warfighters, is united by purpose, mission, Service, and ownership —in other words, it is an end-to-end by Navy/for Navy process. On the other hand, the JS process serves multiple customers (e.g., the COCOMs, the Services). As such, while individual COCOM IPL development processes may be united by mission (though not Service), the final disposition of CGA gaps will be subject to a variety of needs across different missions and purposes.

Bearing these differences in mind—particularly as related to varying end-states—we focused our comparison on steps in both processes that lead to a common milestone of final, prioritized gaps. In the course of the comparison, it became evident that FNC's integration of the solution phase better positions the FNC process to leverage inherent feedbacks between the two phases, thereby enhancing its responsiveness to naval needs.

\section{Recommendations and Strategies for Improvement}

Although solution development activities will remain with the Services, our analysis indicates the JS CGA/PACOM IPL process may benefit from strategies employed by the FNC program, such as communication and comprehensive gap-to-solution tracking strategies. 
In light of this, the study team has identified some key metrics as useful for gauging actual and perceived responsiveness. The first set of metrics relates to determining how well the CGA actually matches and is perceived to match the originally submitted COCOM need. These “Need-to-Gap Development Metrics” focus on measuring gap development activities and include metrics such as the following:

- Number of gaps submitted by COCOMs each year (aggregate and by COCOM),

- Number of COCOM-submitted gaps rejected each year (aggregate and by COCOM), and

- Number of gaps accepted by Joint Staff as-is (aggregate and by COCOM).

The second set of metrics relates to determining whether CGA gaps/needs have been fulfilled or are on a path to fulfillment. These "Gap Fulfillment Metrics" focus on measuring solution development activities and include metrics such as the following:

- Number of CGA gaps with corresponding materiel and non-materiel solution development activities, and

- Number of CGA gaps resolved through solution transition to warfighter.

The team further believes the following recommendations may serve to mitigate some of the frustrations experienced by stakeholders in the JS CGA/PACOM IPL process.

- The JS CGA/PACOM IPL process could expand its boundary to include solution providers. Existing structures (e.g., FCBs, supporting working groups) can be used to facilitate formal participation by the acquisition community. 
- The JS could improve transparency relative to gap status. Formal, accountable, ongoing, and two-way communication will facilitate understanding, expectation management, and feedback.

- Gap and solution progress statistics, collected in coordination with the acquisition and warfighter communities (currently partially tracked by JS and accessible through KMDS), could be documented and published periodically (e.g., annually or bi-annually) and could be actively disseminated to COCOMs and briefed at FCBs and JCBs.

- $\quad$ The JS and PACOM should leverage tracking metrics (as recommended previously) to inform process improvement efforts. The JS process should establish procedures and better define roles and responsibilities to encourage metric and process accountability.

- JS can improve stakeholder satisfaction and commitment by promulgating process steps and clarifying guidance documents. JS should update related Instructions and should consider developing user friendly products and reports that explain the process. 


\section{Reference List}

Assistant Commandant of the Marine Corps; Vice Chief of Naval Operations; Assistant Secretary of the Navy (Research, Development, and Acquisition). (2010, September 7). Technology Oversight Group charter for future naval capabilities [Joint Letter]. Washington, DC: Department of Navy.

Boardman, J., \& Sauser, B. (2008). Systems thinking: Coping with 21st century problems. Boca Raton, FL: CRC Press.

Carlan, K. (2011, March 23). Plans to Resources to Outcomes Process (PROP) [Unpublished PowerPoint presentation].

Chairman of the Joint Chiefs of Staff (CJCS). (2004, December 3). Chairman of the Joint Chiefs of Staff, Combatant Commanders, Chief, National Guard Bureau, and Joint Staff participation in the planning, programming, budgeting and execution process (CJCSI 8501.01A). Retrieved from http://acqnotes.com/Attachments/CJCSI\%208501.01.pdf

Chairman of the Joint Chiefs of Staff (CJCS). (2012, January 10). Joint capabilities integration and development system (CJCSI 3170.01H). Retrieved from http://www.dtic.mil/cjcs_directives/cdata/unlimit/3170_01.pdf

Commander, U.S. Pacific Command (USPACOM). (2011, August 19). Conduct of the Plans to Resources to Outcomes Process (PROP) Within the U.S. Pacific Command (USPACOM). (USPACOMINST 0508.4).

Department of Navy. (2011, February 10). Future Naval Capabilities (FNC) Business Rules [Memorandum]. Washington, DC: Office of Naval Research.

Goldstein, D. (2006, November 8). Future Naval Capabilities Process [Presentation slides]. Retrieved from http://www.ncemt.ctc.com/files/events/proceedings/corrosion20061108/Plenary\%20Sessi on/Goldstein\%20-\%20FNC\%20Process-1.pdf

Office of Naval Research. (n.d.a). Future Naval Capabilities (FNCs). Retrieved from http://www.onr.navy.mil/Science-Technology/Directorates/Transition/Future-NavalCapabilities-FNC.aspx 
Office of Naval Research. (n.d.b). Future Naval Capabilities. Retrieved from http://www.onr.navy.mil/en/Science-Technology/Departments/Code-35/AllPrograms/air-warfare-352/Future-Naval-Capabilities.aspx

Office of Naval Research. (2011, February 1). Future Naval Capabilities Program [PowerPoint slides].

Office of Naval Research. (2012, March 9). Future Naval Capabilities [PowerPoint slides disseminated during FNC training course at FNC Program Office].

Under Secretary of Defense for Acquisition, Technology, and Logistics (USD[AT\&L]). (2008, December 8). Operation of the Defense Acquisition System (DoD Instruction 5000.02). Washington, DC.

U.S. Naval Research Laboratory. (n.d.). Future Naval Capabilities. Retrieved from http://www.nrl.navy.mil/research/future-naval-capabilities/ 


\section{Acknowledgements}

This material is based upon work supported by the Naval Postgraduate School Acquisition Research program under Grant No. N00244-12-1-0016.

We gratefully thank Steve Smolinski (FNC Program Director) and members of his staff for extending their support and time. They provided critical information and discussions regarding the United States Navy’s FNC program.

Special thanks also go to Colonel Keith Duffy, Chief, Planning and Integration Branch, Joint Capability Division, J-8, Joint Staff; Captain Dave Glenister, Planning and Integration Branch, Joint Capability Division, J-8, Joint Staff; Mr. Ken Bruner, Science and Technology Advisor, PACOM; Mr. Kit Carlan, Future Capabilities Analyst, J-82, PACOM for key information and helpful discussions on the Joint Staff Capability Gap Assessment and PACOM’s IPL process.

This study is a culmination of the independent analyses by the authors and does not represent the views or positions of the acknowledged individuals or organizations. 


\section{Jaime Frittman}

\section{About the Authors}

Ms. Frittman is a technical manager of the Applied Systems Thinking Consulting Group within Analytic Services Inc. Jaime has a background in emergency management, chemical and biological defense, systems engineering, and systems thinking. Her acquisition-related experience includes positions working Joint Service modernization programs. In addition to this, she has conducted and/or overseen a multitude of projects related to the application of systems thinking tools and methods to problems in a variety of technical domains. She has a master’s degree in systems engineering from Stevens Institute of Technology. Contact Info: 2900 South Quincy Street, Arlington, VA 22206. Tel: (703) 416-3451, jaime.frittman@anser.org

\section{Sibel McGee}

Dr. Sibel McGee is a principal analyst at Analytic Services Inc. and an adjunct assistant professor at University of Maryland University College. Her interest areas include systems thinking, politics of culture, terrorism, radicalization, homeland security, emergency management, and public resilience. She is the managing editor of Journal of Homeland Security and Emergency Management. She received a BS degree from Middle East Technical University and an MS degree from the University of Bonn. She holds a PhD in political science from Texas A\&M University. Contact Info: 2900 South Quincy Street, Arlington, VA 22206. Tel: (703) 416-3342, Fax: (703) 416- 1367, sibel.mcgee@anser.org 


\section{John Yuhas}

Mr. Yuhas is a project manager and principal analyst at Analytic Services Inc. with government and industry experience in defense research, development, acquisition, and operations. His experience covers intelligence, forensics, biometrics, rotary wing, logistics, C4ISR, and training. He has been instrumental in developing management, organization, and transition strategies/plans. Mr. Yuhas has chaired or served as a member of U.S. and international committees and joint working groups. He previously held positions with industry; USF-I in Baghdad, Iraq; OSD(ATL); U.S. Army (ALT); and Army Aviation Systems Command. Contact Info: 2900 South Quincy Street, Arlington, VA 22206. Tel: (703) 416-8488,

john.yuhas@anser.org 